\title{
Towards Personalized Intensive Care Decision Support Using a Bayesian Network: A Multicenter Glycemic Control Study
}

\author{
Asma Abu-Samah ${ }^{1}$, Normy Norfiza Abdul Razak ${ }^{2}$, Fatanah Mohamad Suhaimi ${ }^{3}$, \\ Ummu Kulthum Jamaludin ${ }^{4}$, and James Geoffrey Chase ${ }^{5}$ \\ ${ }^{1}$ Institute of Energy Infrastructure, Universiti Tenaga Nasional / Selangor, 43650, Malaysia Asma@uniten.edu.my \\ ${ }^{2}$ Department of Electronics \& Communication, Universiti Tenaga Nasional / Selangor, 43650, Malaysia \\ ${ }^{3}$ Advanced Medical \& Dental Institute, Universiti Sains Malaysia / Penang, Malaysia \\ ${ }^{4}$ Faculty of Mechanical Engineering, Universiti Malaysia Pahang / Pahang, Malaysia \\ ${ }^{5}$ Department of Mechanical Engineering, University of Canterbury / Christchurch, New Zealand \\ * Corresponding Author: James Geoffrey Chase
}

Received March 31, 2019; Revised April 26, 2019; Accepted May 28, 2019; Published June 30, 2019

${ }^{*}$ Regular Paper

* Extended from a Conference: Preliminary results of this paper were presented at the IEEE TENCON2018. This paper has been accepted by the editorial board through the regular reviewing process that confirms the original contribution.

Abstract: Personalized treatment in glycemic control (GC) is a visibly promising research area that requires improved mechanisms providing patient-specific procedures to enable complicated decision support. Available per-patient data must be more than written records, and be fully integrated in this personalization process. This article presents a process for relating the intensive care unit patients' demographic and admission data to their GC performance. With this objective, a probabilistic Bayesian network was chosen to provide more personalized decisions. As a case study, average daily blood glucose measurements were chosen as the interest target node in order to weigh GC that provides a reduced nursing workload. To test the idea, data from 482 patients, with nine variables from four Malaysian intensive care units with different controls were exploited. The identified steps crucial in building a dependable model are variable selection, continuous state discretization, and unsupervised structure learning. Using a multi-target node evaluation, a network with $80 \%$ mean overall classification precision was obtained with a normalized equal distance discretization algorithm and a maximum weight spanning tree technique. Meanwhile, the interest target node scored $90.39 \%$ precision. The results from this study, which are complemented with an evaluation of missing data, are proposed as a benchmark for using Bayesian networks in this type of application.

Keywords: Personalized glycemic control, Intensive care units, Bayesian network, Discretization technique, performance prediction

\section{Introduction}

Since the birth of intensive care medicine, researchers have tended to study cause-and-effect results on patients with similar signs and symptoms [1-4]. As health informatics and awareness of improved intensive care medicine has progressed, an increasingly personalized medicinal approach is entering intensive care units (ICUs) [5-7]. The approach considers integrating individual characteristics to recommend optimized patient-specific treatment. For example, an increasing number of ICUs are starting to use validated and computerized algorithms with patient-specific physiological models, such as EndoTool, LOGIC-Insulin, stochastic targeted (STAR), and Glucospace [8-14]. These approaches are based on a closed-loop control system with continuous glucose monitoring and computer-assisted titration of insulin on glucose measurements to minimize hyperglycemia.

The approaches improve clinical guidelines based on each patient's metabolic profile, minimize hypoglycemia, and reduce nursing staff workload. However, none systematically considers per-patient demographic 
Table 1. Summary of Data.

\begin{tabular}{|c|c|c|c|c|}
\hline & HTAA & UMMC & HUSM & IIUMMC \\
\hline Type of control & \multicolumn{3}{|c|}{ Rule-based sliding scale approach } & $\begin{array}{c}\text { Computerized and patient-specific } \\
\text { approach }\end{array}$ \\
\hline Target range [in mmol/L] & $5.1-8.0$ & $6.0-9.0$ & $6.0-10.0$ & $6.0-10.0$ \\
\hline Total number of patients & \multirow[b]{2}{*}{210} & 90 & 15 & \multirow[b]{2}{*}{158} \\
\hline $\begin{array}{l}\text { Number of patients with } \\
\text { complete data }\end{array}$ & & 10 & 7 & \\
\hline Age [IQR, years] & $56.5[45.0-64.0]$ & $62.0[55.3-63.8]$ & $55.5[50.0-60.25]$ & $56.0[62.0-68.0]$ \\
\hline
\end{tabular}

background and admission conditions, such as age, height, weight, and comorbidities, and judgments are often left to expert opinion [15]. Furthermore, despite the growing amount of computerized patient-specific control, a majority of hospitals around the world, especially in less developed countries, still use manual sliding scales based on generalized rules to control glycemia, such as in Malaysian ICUs [16]. In any case, all these ICU data need to be exploited to make the best decisions for critically ill patients. Instead of regular measurement, some of these data are one-time information and do not have any patterns to signal patient variability; nevertheless, they may add value that can support and enhance clinical decision support, such as controls that optimize workload.

The Bayesian network [17, 18], a probabilistic and graphical model often used to model uncertainty and causality, offers a potential solution with applications ranging from medical diagnosis [19], detection [20], and prediction [21] as well as decision-making systems [22]. Due to their directed graphical structure, Bayesian networks (BNs) are intuitively interpretable, thus assisting and expediting human decision support in ways generalized machine learning cannot. In essence, the BN structure helps "explain" its output. A BN provides an efficient factorization of the joint probability distribution over a set of random variables. Patient-specific data that often contain a combination of discrete and continuous variables, can be deployed in structured learning and inference to perform diagnosis, prognosis, or to simply find the causal relationship between variables. Bayesian networks offer efficient parameter learning with relatively few learning data, and offer an ideal framework to deal with missing values.

The objective of this paper is to study the integration of demographic and admission data (one-off input values from multiple medical center ICUs) with glycemic control performance, and to try and interpret the possible relationships between the variables. As a case study, this paper works on one interest target node (set as the average number of daily blood glucose measurements) to explore the feasibility of choosing optimized nursing intervention based on patient background. From this viewpoint, the paper focuses on the choices of discretization techniques towards selected data (which are one-off but with continuous values), on structured learning steps, and on inference in cases of missing data.

\section{Methods}

\subsection{Glycemic Control Data}

The study was conducted on data from 482 critically ill Malaysian ICUs patients from the Hospital Tengku Ampuan Afzan (HTAA), the Universiti Malaya Medical Centre (UMMC), the Hospital Universiti Sains Malaysia (HUSM), and the International Islamic University Malaysia Medical Centre (IIUMMC). Patients were treated under four different controls: three sliding-scale insulin infusion approaches (324 patients), and one computerized STAR protocol (158 patients).

(1) HTAA, UMMC, and HUSM Sliding Scale Approaches: In this protocol, the medical staff performs treatment based on rules [16] to maintain glycemic levels within a chosen target range. Specific insulin infusion values are proposed solely based on associated BG-level measurements. In these ICUs, the BG target range is 5.1$8.0,6.0-9.0$ or $6.0-10.0 \mathrm{mmol} / \mathrm{L}$. BG monitoring and treatment are performed hourly after administering insulin has started. When there is no requirement for an insulin rate change for two consecutive hours, $\mathrm{BG}$ is then measured every two to four hours. Monitoring frequency is reduced once the patient is considered stable.

(2) IIUMMC STAR control: STAR is an automated glycemic control protocol that characterizes and forecasts changes in per-patient metabolic state. It is more personalized in the sense that insulin infusion values depend on each and every patient's insulin sensitivity variations, but it does not capture the patient's background (age, gender, diabetes history, etc.). It is designed to be used in real-time bedside care with a computer tablet. Its predictions are based on a stochastic model over the one to three hours subsequent to potential variations in patientspecific insulin sensitivity [23-25]. STAR is a solution to the personalized approach and has shown promising results. It is the default treatment in Christchurch, New Zealand, and Gyula, Hungary, hospital ICUs [26]. Since December 2016, it has been implemented in the IIUMMC ICU in Malaysia as part of a Malaysian pilot trial [27, 28]. The flexibility of STAR includes BG-level target range, measurement frequency, patient safety within a predefined desired hypoglycemia risk, and local nutrition practices $[13,29]$. The data from this ICU came from the 6.0-10.0 $\mathrm{mmol} / \mathrm{L}$ target range. STAR, however, does not include demographic data in the control feedback. 
and their control approach.

\subsection{Bayesian Network}

A Bayesian network models a variable as a node, and the potential causal relationship between two variables as a directed arc. These create the $\mathrm{BN}$ structure, and to complete it, a conditional probability table (CPT) is assembled for each node to represent the probabilities of each value of a node, given the conditions of its parents. The structure, along with the CPT, can be built from human knowledge, machine-learned from training datasets, or from a combination of both. This study proposes that both be used in order to learn from data and testing over random data sampling.

While BNs can be trained from continuous variables directly, it is common to discretize the variables into states to prepare a dataset that enables the Bayesian network structure's learning. The purpose is to minimize computation speed by avoiding having to consider variable-interaction complexities. In discrete-state cases (and provided the probability of an event, B, is not zero), Bayes theorem is used to relate the conditional and marginal probabilities of two events, A and B, (Eq. (1)):

$$
\mathrm{P}(\mathrm{A} \mid \mathrm{B})=\frac{\mathrm{P}(\mathrm{B} \mid \mathrm{A}) \cdot \mathrm{P}(\mathrm{A})}{\mathrm{P}(\mathrm{B})}
$$

- $\mathrm{P}(\mathrm{A} \mid \mathrm{B})$ is the conditional probability of $\mathrm{A}$, given $\mathrm{B}$. It is also called the posterior probability.

- $\mathrm{P}(\mathrm{B} \mid \mathrm{A})$ is the conditional probability of $\mathrm{B}$, given $\mathrm{A}$. It is also called the likelihood.

- $\mathrm{P}(\mathrm{B})$ is the prior or marginal probability of $\mathrm{B}$, and acts as a normalizing constant

The choice of discretization technique has a striking impact on the network's classification and prediction precision and computation speed, and on the interpretability of the resulting network. For the purpose of this study, discretization for each continuous variable was limited to between three states only.

The study was conducted using a tool known as BayesiaLab (version 7.0) to generate the Bayesian networks. BayesiaLab is a complete tool for the creation and use of Bayesian networks. It provides nine discretization techniques. For our study, comparisons were made only between K-means, density approximation, equal distance, normalized equal distance, and equal frequency. The structure-learning step follows, and was performed using unsupervised leaning algorithms paired with score-based learning algorithms to discover all the probabilistic relations in the data. The available unsupervised learning algorithms were maximum weight spanning tree (MWST), Taboo, Taboo Order, Equivalent Class (EQ) and SopLEQ. As opposed to the constraintbased algorithms that use independence tests to add or remove arcs between nodes, the minimum description length score [30] or Pearson correlation score were deployed to measure the quality of network candidates corresponding to the available datasets.

\subsection{Multi-target Node Evaluation}

The resulting networks' performance evaluations were done using a multi-node target analysis system. The basis is to consider each node in the network as a target node, and final performance is based on overall performance. For each node as a target, the quantitative performance of all candidates was evaluated using the test dataset with the following three metrics: (i) overall precision, (ii) root mean square error (RMSE), and (iii) overall calibration index. Overall precision characterizes the power of classification; the calibration index estimates the posterior probability distribution of the target node; and RMSE forecasts a numerical value using a posterior probability distribution to compute the weighted average of the target node's value.

\subsection{Missing-data Completion}

Out of the 482 sets of data, 97 were missing the patient's height owing to the complication of measuring a critically-ill patient's height upon admission to the ICU. This seems to be common, and the 97 sets came from HUSM and UMMC. Data from HTAA were only from patients with complete datasets, and the STAR protocol obliges users to enter both height and weight upon the start of the control. These patients' data will be injected in the resulting Bayesian network to see the prediction performance.

\section{Results and Discussion}

By using Bayesian networks as the framework and BayesiaLab as the tool, the process began with the identification of variables to be used in the modelling. Common variables that were available from the four ICUs provided data, using four different glycemic controls (GC). Ten variables were extracted: type of control, age, gender, height, weight, diabetes mellitus and hypertension status upon admission to the ICU, initial BG level, total hours under GC treatment, and number of measurements involved. The last two variables were then merged and converted into a single variable (average daily measurement) to measure the nursing workload as the main prediction interest.

These variables can be classified into two categories; i) patient background and ii) glycemic control information. Amongst these variables, type of control, gender, and diabetes mellitus and hypertension status are discrete and binary. All the others need to be discretized before being used in the model. The summary of variables is presented in Table 2.

Due to the nature of ICUs, patient height upon admission is sometimes difficult to measure. Some patient data from HUSM and UMMC that were without the patient's height were considered missing data cases. Two datasets were then created with the nine variables, one with 385 complete sets of data, and another including the 97 patients with missing heights. Generated results are presented and discussed based on the final networks with best prediction score first, and then the discretization 
Table 2. Summary of Variables.

\begin{tabular}{|c|c|c|c|}
\hline \hline No. & Category & Variables & Type \\
\hline 1 & Glycemic control info & Type of control & Discrete: HTAA, IIUM, HUSM, UMMC \\
\hline 2 & Patient background & Age & Continuous: (years) \\
\hline 3 & Patient background & Gender & Binary: $\mathrm{m} / \mathrm{f}$ \\
\hline 4 & Patient background & Height & Continuous: $(\mathrm{cm})$ \\
\hline 5 & Patient background & Weight \\
\hline 6 & Patient background & Diabetes mellitus & Binary: $0 / 1$ \\
\hline 7 & Patient background & Hypertension & Binary: $0 / 1$ \\
\hline 8 & Glycemic Control info & Initial BG level & Continuous: $(\mathrm{mmol} / \mathrm{L})$ \\
\hline 9 & Glycemic Control info & Average daily measurement & Continuous: $(\mathrm{n})$ \\
\hline
\end{tabular}

Table 3. Summary of Performance in Terms of Overall Precision for Target Nodes using Different Discretization Techniques.

\begin{tabular}{|c|c|c|c|c|c|}
\hline \hline Overall precision & K-means & $\begin{array}{c}\text { Density } \\
\text { approximation }\end{array}$ & $\begin{array}{c}\text { Normalized equal } \\
\text { distance }\end{array}$ & $\begin{array}{c}\text { Equal } \\
\text { distance }\end{array}$ & $\begin{array}{c}\text { Equal } \\
\text { frequency }\end{array}$ \\
\cline { 2 - 7 } & 74.08 & 69.90 & 80.00 & 79.80 & 65.71 \\
\hline Mean (\%) & 11.24 & 14.18 & 7.98 & 11.18 & 15.89 \\
\cline { 1 - 6 } Standard deviation (\%) & $\begin{array}{c}55.06 \% \\
\text { (age) }\end{array}$ & $\begin{array}{c}43.90 \% \\
\text { (age) }\end{array}$ & $\begin{array}{c}60.52 \% \\
\text { (age) }\end{array}$ & $\begin{array}{c}60.52 \% \\
\text { (age) }\end{array}$ & $\begin{array}{c}43.38 \% \\
\text { (initial BG) }\end{array}$ \\
\hline Minimum (\%) & $\begin{array}{c}90.65 / \\
\text { (type of } \\
\text { control) }\end{array}$ & $\begin{array}{c}89.10 / \\
\text { (type of control) }\end{array}$ & (interest target node*) & $\begin{array}{c}93.51 / \\
\text { (Height) }\end{array}$ & $\begin{array}{c}85.45 / \\
\text { (Type of } \\
\text { control) }\end{array}$ \\
\hline On interest target node* (\%) & 85.45 & 80.26 & 90.39 & 90.39 & 67.01 \\
\hline
\end{tabular}

*Interest node: The average daily blood glucose measurement

algorithms that produced them. The comparative performance between networks using different discretization techniques can be seen in Table 3. Minimum mean precision was $69.90 \%$. Based on the results, BNs built from the normalized equal distance displays the best compromise of classification performance: $80 \%$ mean precision with $7.98 \%$ standard deviation. Its maximum precision was designated to the interest target node (average daily measurement at $90.39 \%$ precision). Other techniques followed with type of control and height nodes with maximum precision.

The equal distance and normalized equal distance techniques performed slightly differently. Equal distance directly discretizes equal distances based on the range of the variable, whereas normalized equal distance first uses a smoothing algorithm to clean the outliers, and then computes the equal distances. The higher standard deviation and maximum overall precision from the equal distance technique indicates there were outliers in the data. Comparisons of discretization intervals between the two datasets indicate that only height had different sets of intervals, and indicate that outliers come from this variable. The differences between the two intervals can be seen in Table 4. Fig. 1 displays the normal probability distribution of each node of the network and its values, allowing us to see the discretization intervals with the associated initial distribution. With the prevalence of data coming from HTAA and IIUMMC, $53.25 \%$ of the patients had diabetes, and $56.10 \%$ had a history of hypertension. What could not be captured in the distribution is the fact that $34.29 \%$ of patients had both morbidities.
Table 4. Discretization Interval between the Equal Distance and Normalized Equal Distance Techniques.

\begin{tabular}{|c|c|c|}
\hline \multirow{2}{*}{ Node } & Equal distance & $\begin{array}{c}\text { Normalized equal } \\
\text { distance }\end{array}$ \\
\cline { 2 - 3 } & $<=60-102 \mathrm{~cm}$ & $<=60-130 \mathrm{~cm}$ \\
\cline { 2 - 3 } Height & $<102-144 \mathrm{~cm}$ & $<130-158 \mathrm{~cm}$ \\
\cline { 2 - 3 } & $<144-186$ & $<158-186$ \\
\hline
\end{tabular}

The resulting $\mathrm{BN}$ from the normalized equal distance discretization technique is presented in Fig. 2. Nodes are colored according to their mutual relationship strength. The network was generated using the unsupervised MWST approach that uses Pearson correlation coefficients for every pair of nodes. These coefficients are then used as weights to build a network maximizing the total sum of their squared values. MWST using Pearson correlation, and not using minimum description length (MDL) score as an objective function, is the only technique consistently providing a final network with all nodes connected to each other. This result is explained by the fact that MDL scores consider the correlation plus the structural complexity of the network, thus establishing automatic significance thresholds. However, the Pearson correlation is only based on correlation, without any significance threshold. Thus, it always returns networks in which all the nodes are connected, even in the case of very weak relationships, which may not be accurate or desired. Other techniques (Taboo, Taboo Order, Equivalent Class [EQ] and SopEQ) paired with either MDL or the Pearson score provided 


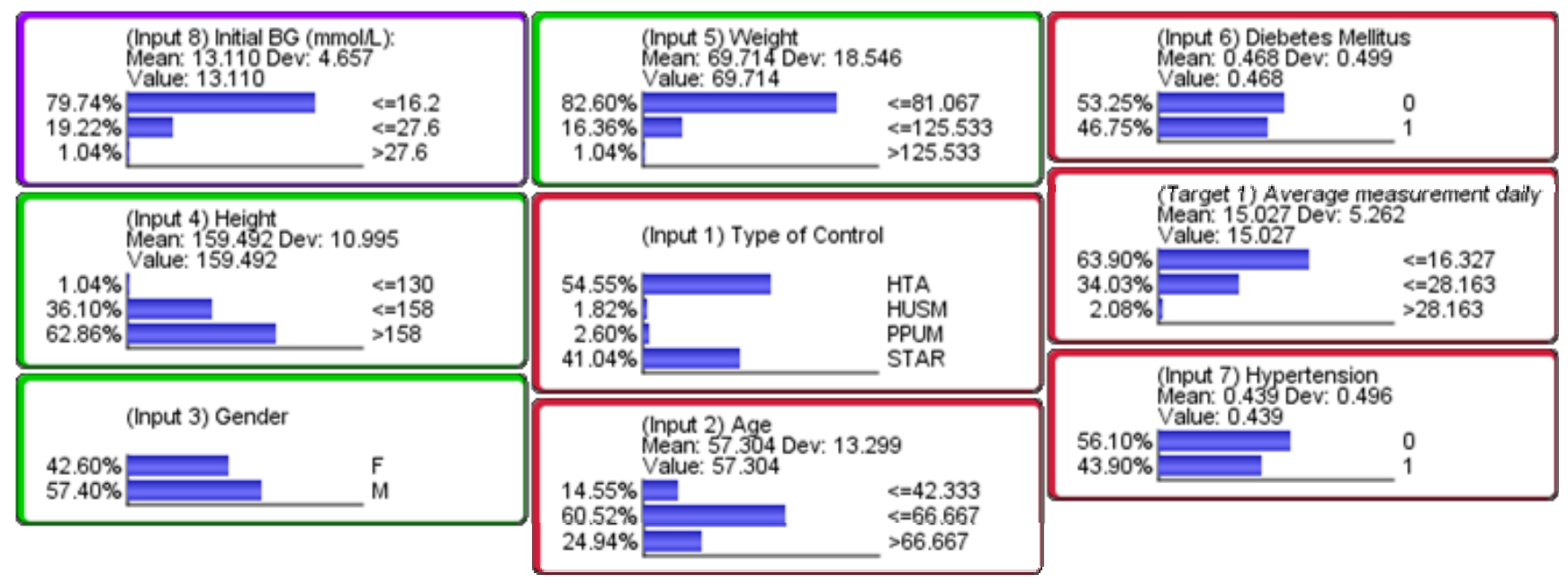

Fig. 1. Resulting Bayesian network probability distribution.

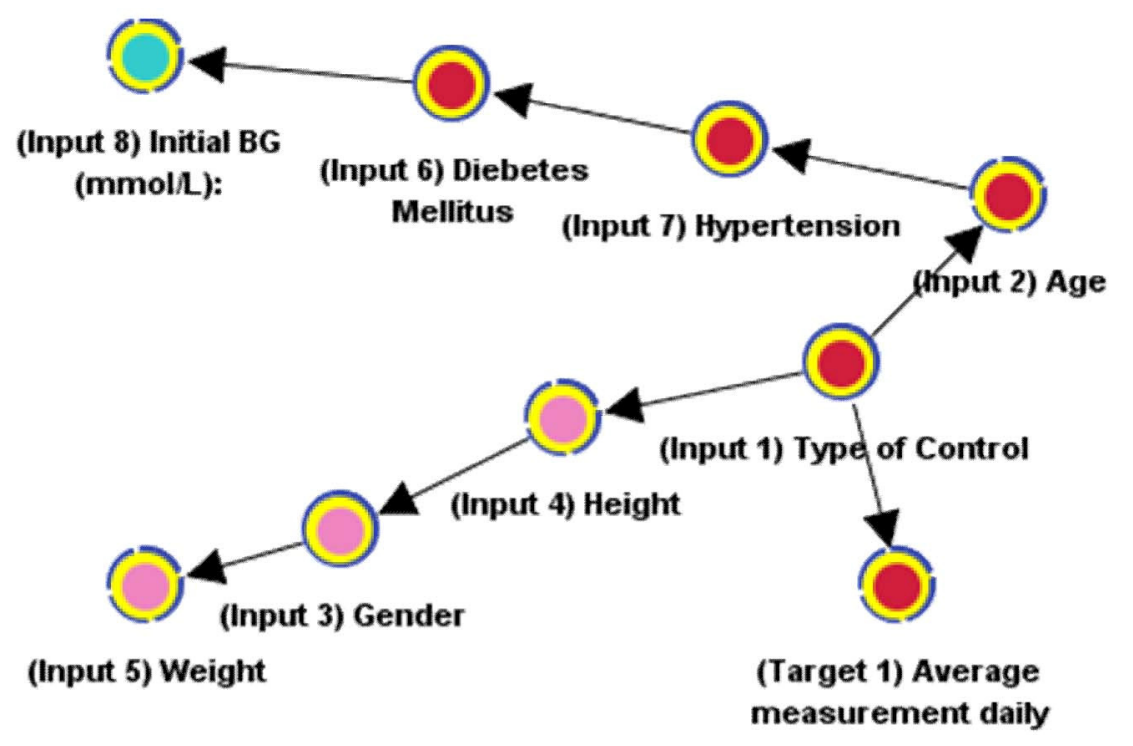

Fig. 2. Bayesian network using normalized equal distance discretization and maximum weight spanning tree structure learning.

networks with only two nodes connected.

Qualitatively, the network reveals type of control as the central node directly correlated to age, average daily measurement, and height. In terms of patient background, height, weight and gender are clustered together on one side of the type of control. Diabetes mellitus and hypertension are clustered with age on the other side. The initial BG is the most distanced node in the network. The proximity of hypertension status to average daily measurement, if compared to diabetes mellitus status, signals prioritizing investigation and potential incorporation of hypertension status or parameters in modelling the overall approach for optimized or efficient glucose control. Second, the arrows in the generated BN with the MWST structure learning based on the Pearson correlation only signifies the strength of the correlation between two nodes, and not necessarily the causal relationship between them. Even if the arcs change orientation, the prediction results stay the same. This network enables the prediction of average daily measurement given information from any of the other nodes. For example, if a patient is known to be male, age 70 , with both diabetes and hypertension, the resulting inference for average daily measurement is $<16.327$ times if using an HTAA control, but falls to between 16.327 and 28.163 times if using STAR.

To further elaborate on the result from the network, Table 4 presents the RMSE and overall calibration index. RMSE is smaller for nodes with minimum classification precision, and $41 \%$ larger for the maximum. This means, while the interest target node has a higher classification power, the forecasting of its continuous values posterior probability distribution is low. Results are also compared with prediction using the missing-data datasets from UMMC and HUSM on the resulting network to evaluate the ability of the Bayesian network as a classifier under the circumstances. The results complemented Table 4. Interestingly, the mean loss of overall prediction only amounts to $2.5 \%$. Despite a bigger standard deviation, the maximum precision with the target node is higher, at 
Table 4. Multi-Target Discretization from Three Normalized Equal Distance Discretization Techniques.

\begin{tabular}{|c|c|c|c|}
\hline Metrics & $\begin{array}{c}\text { Overall } \\
\text { precision }\end{array}$ & RMSE & $\begin{array}{c}\text { Overall calibration } \\
\text { index }\end{array}$ \\
\hline \multicolumn{4}{|c|}{ With the original dataset (385 patients) } \\
\hline Mean & $80.00 \%$ & 5.49 & $80.04 \%$ \\
\hline Standard deviation & $7.98 \%$ & 5.96 & $8.36 \%$ \\
\hline $\begin{array}{l}\text { Minimum } \\
\text { (node: age) }\end{array}$ & $60.52 \%$ & 0.38 & $67.71 \%$ \\
\hline $\begin{array}{l}\text { Maximum (node: } \\
\text { average workload) }\end{array}$ & $90.39 \%$ & 17.67 & $93.89 \%$ \\
\hline \multicolumn{4}{|c|}{ With missing-data dataset (482 patients) } \\
\hline Mean & $77.50 \%$ & 5.69 & $74.08 \%$ \\
\hline Standard deviation & $8.50 \%$ & 6.08 & $4.56 \%$ \\
\hline $\begin{array}{l}\text { Minimum } \\
\text { (node: age) }\end{array}$ & $59.00 \%$ & 0.40 & $66.86 \%$ \\
\hline $\begin{array}{l}\text { Maximum (node: } \\
\text { average workload) }\end{array}$ & $92.05 \%$ & 17.67 & $81.75 \%$ \\
\hline
\end{tabular}

92.05\%. However, the overall calibration index is lower, at $81.57 \%$, and this is attributed to the missing values, which impacted the computation of the posterior probability of the cohort's network. Even in this case, the results show that this network can provide reliable classification, even with missing heights, and serves as the objective to help doctors decide on the control based on per-patient background and predicted average daily measurement.

\section{Conclusion}

The Bayesian network, even in its simplest form, has three major advantages. It explicitly provides the conditional probability distributions of the values of a class attribute, given the values of the other input attributes; it has the ability to deal with missing values, and finally, the graphical network model is easy to understand. For these reasons, the awareness to apply and use the Bayesian network causality property in the medical context is increasing. This paper presented results from linking data coming from personalized patient backgrounds and their glycemic control treatment in the ICU using a Bayesian network. It focused on the structural machine-learned process that potentially gains some relevance for future research and practical applications for BNs in ICUs. The performance of the BN was compared within its building algorithms, such as discretization and the unsupervised structure learning process. The study is complemented with an assessment of the resulting BN network prediction with a dataset missing some of the data.

The study revealed that many discretization techniques support the learning of a Bayesian network from these types of data, and classification statistical validity provides excellent prediction. The resulting network can also be used in cases with a missing height, which occur regularly in critical care units. However, with the current study design, only the maximum weight spanning tree approach that uses Pearson correlation coefficients consistently gives the best overall performance when this type of network cannot indicate causality per our initial interest. A larger-scale study with different combinations of datasets needs to be done to achieve all the above, but with a learning technique that considers causality as an end network.

\section{Acknowledgement}

The authors thankfully acknowledge Hospital Tengku Ampuan Afzan (HTAA), Universiti Malaya Medical Centre (UMMC), Hospital Universiti Sains Malaysia (HUSM) and International Islamic University Malaysia Medical Centre (IIUMMC) ICUs' clinical teams for their support and provision of data for this study. The authors also acknowledge the FRGS Grant from the Malaysian Ministry of Higher Education (MOHE), the BOLD grant, and UNITEN for their roles in the ongoing research.

\section{References}

[1] T-K. Nfor et al., "The impact of organ failures and their relationship with outcome in intensive care: analysis of a prospective multicentre database of adult admissions," Anaesthesia, 61(8), pp. 731-738, 2006. Article (CrossRef Link)

[2] P. Dennen et al., "Acute kidney injury in the intensive care unit: an update and primer for the intensivist," Critical care medicine, 38(1), pp. 261275, 2010. Article (CrossRef Link)

[3] J. Clain et al., "Glucose control in critical care," World journal of diabetes, 6(9), pp. 1082, 2015. Article (CrossRef Link)2

[4] R-S-Y.Wong et al., "An application of Bayesian approach in modeling risk of death in an intensive care unit," PloS one, 11(3), pp. e0151949, 2016. Article (CrossRef Link)

[5] M-M. Ghassemi et al., "A data-driven approach to optimized medication dosing: a focus on heparin," Intensive care medicine, 40(9), pp. 1332-1339, 2014. Article (CrossRef Link)

[6] A-E. Johnson et al., "Machine learning and decision support in critical care," Proceedings of the IEEE, Institute of Electrical and Electronics Engineers, 104(2), pp. 444, 2016. Article (CrossRef Link)

[7] J-G. Chase et al., "Next-generation, personalised, model-based critical care medicine: a state-of-the art review of in silico virtual patient models, methods, and cohorts, and how to validation them," Biomedical engineering online, 17(1), pp. 24, 2018. Article (CrossRef Link)

[8] S. Cochran et al., "ENDOTOOL Software for Tight Glucose Control for Critically Il Patients 260," Critical Care Medicine, 34(12), pp. A68, 2006.

[9] N-M. Saur et al., "Software-Guided Insulin Dosing: Tight Glycemic Control and Decreased Glycemic Derangements in Critically Ill Patients," in Mayo Clinic Proceedings, Vol. 88, No. 9, pp. 920-929, Elsevier, 2013. Article (CrossRef Link) 
[10] T. Van Herpe et al., "LOGIC-Insulin Algorithm Guided versus Nurse-Directed Blood Glucose Control during Critical Illness: the LOGIC-1 SingleCenter, Randomized, Controlled Clinical Trial," Diabetes Care, 36(2), pp. 188-194, 2013. Article (CrossRef Link)

[11] A. Evans et al., "Pilot Proof of Concept Clinical Trials of Stochastic Targeted (STAR) Glycemic Control," Annals of Intensive Care, 1(1), pp. 38, 2011. Article (CrossRef Link)

[12] A. Evans et al., "Stochastic Targeted (STAR) Glycemic Control: Design, Safety, and Performance," Journal of Diabetes Science and Technology, 6(1), pp. 102-115, 2012. Article (CrossRef Link)

[13] J. Blaha et al., "Space GlucoseControl system for blood glucose control in intensive care patients-a European multicentre observational study". $B M C$ anesthesiology, 16(1), pp. 8. 2015. Article (CrossRef Link)

[14] B. Xu et al., "Comparison of Space Glucose Control and Routine Glucose Management Protocol for Glycemic Control in Critically Ill Patients: A Prospective, Randomized Clinical Study," Chinese medical journal, 130(17), 2041. Article (CrossRef $\underline{\text { Link) }}$

[15] J-G. Chase et al., "Impact of Human Factors on Clinical Protocol Performance: a Proposed Assessment Framework and Case Examples," Journal of Diabetes Science and Technology, 2(3), pp. 409-416, May. 2008. Article (CrossRef Link)

[16] Kementerian Kesihatan Malaysia, Malaysian Society of Intensive Care, and Malaysia Society of Intensive Care, "Management Protocols In ICU Malaysia," 186, September 2012". Article (CrossRef Link)

[17] J. Pearl, "Probabilistic Reasoning in Intelligent Systems: Networks of Plausible Inference," Elsevier, 28, June. 2014.

[18] D. Koller, and N. Friedman, "Probabilistic Graphical Models: Principles and Techniques," MIT Press, 2009.

[19] J-L. Lustgarten et al., "Application of an Efficient Bayesian Discretization Method to Biomedical Data," BMC Bioinformatics, 12(1), pp. 309, 2011. Article (CrossRef Link)

[20] S-K. Nachimuthu, and P-J. Haug, "Early Detection of Sepsis in the Emergency Department using Dynamic Bayesian Networks," In AMIA Annual Symposium Proceedings, American Medical Informatics Association, 2012. Article (CrossRef Link)

[21] D. Chung et al., "General Bayesian Network Approach to Health Informatics Prediction: Emphasis on Performance Comparison," Procedia-Social and Behavioral Sciences, 28(81), pp. 465-468, June, 2013. Article (CrossRef Link)

[22] B. Thanathornwong, "Bayesian-Based Decision Support System for Assessing the Needs for Orthodontic Treatment," Healthcare Informatics Research, 24(1), pp. 22-28, January. 2018. Article (CrossRef Link)

[23] J. Lin et al., "Stochastic Modelling of Insulin Sensitivity and Adaptive Glycemic Control for
Critical Care," Computer Methods and Programs in Biomedicine, 89(2), pp. 141-152, 2008. Article (CrossRef Link)

[24] J. Lin et al., "A physiological Intensive Control Insulin-Nutrition-Glucose (ICING) Model Validated in Critically Ill Patients," Computer Methods and Programs in Biomedicine, 102(2), pp. 192-205, 2011. Article (CrossRef Link)

[25] A-J. Le Compte et al., "Blood Glucose Prediction using Stochastic Modeling in Neonatal Intensive Care," IEEE Transactions on Biomedical Engineering, 57(3), pp. 509-518, 2010. Article (CrossRef Link)

[26] K-W. Stewart et al., "Safety, Efficacy and Clinical Generalization of the STAR Protocol: a Retrospective Analysis," Annals of Intensive Care, 6(1), pp.24, 2016. Article (CrossRef Link)

[27] A. Abu-Samah et al., "Model-Based Insulin-Nutrition Administration for Glycemic Control in Malaysian Critical Care: First Pilot Trial," in International Conference for Innovation in Biomedical Engineering and Life Sciences, Springer, Singapore, December. 2017. Article (CrossRef Link)

[28] A. Abu-Samah et al., "Model-based glycemic control in a Malaysian intensive care unit: performance and safety study," Medical Devices: Evidence and Research, 12, pp. 216-226, 2019. Article (CrossRef Link)

[29] L-M. Fisk et al., "STAR Development and Protocol Comparison," IEEE Transactions on Biomedical Engineering, 59(12), pp. 3357-3364, 2012. Article (CrossRef Link)

[30] W. Lam, and F. Bacchus, "Learning Bayesian Belief Networks: An Approach based on the MDL Principle," Computational Intelligence, 10(3), pp. 269-293, 1994. Article (CrossRef Link)

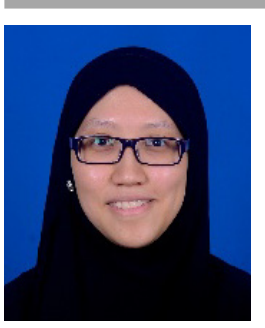

Asma Abu-Samah is a post-doctoral researcher of Automated Control applied to Bio-Medical Engineering at the National Energy University (UNITEN), Malaysia. She received her B.Eng. and M.Eng. in Control Systems of Electrical Energy from Université de Joseph Fourier, France, in 2008 and 2010, respectively, and her PhD in Automated Control and Production Systems from the Université Grenoble Alpes in 2016. Dr. Asma served, or currently serves, as a reviewer for many Journals, Conferences, Symposiums, and BioMedical Workshops as well as in the Prognostics and Health Management area. Her research interests include data-based failure prediction and diagnosis in industrial equipment as well as the human physiological system. 


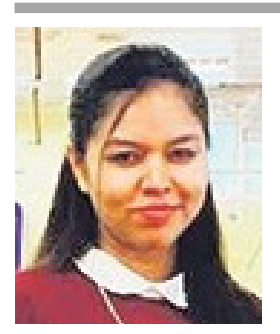

Normy Norfiza Abdul Razak is a senior lecturer with the Department of Electronics \& Communication Engineering, National Energy University (UNITEN), Malaysia. She received her B.Eng. in Electrical Electronics from UNITEN, and an MSc in Electrical Engineering-Control and Automation from the University of Nottingham, in 2003 and 2014, respectively. She completed her $\mathrm{PhD}$ in Bio-EngineeringComputational Modeling and Physiological Control from the University of Canterbury in 2011. She is currently the director of the UNITEN Research Management Centre.

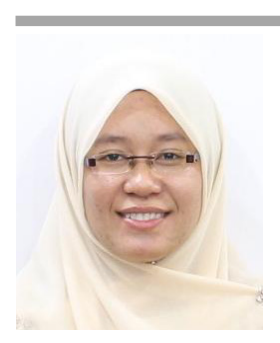

Fatanah Mohamad Suhaimi is a senior lecturer at the Advanced Medical and Dental Institute (AMDI) of Universiti Sains Malaysia (USM). She received a B.Eng. in Mechatronics Engineering (Honors) from the International Islamic University Malaysia. In 2012, she was awarded a $\mathrm{PhD}$ in Mechanical Engineering from the University of Canterbury, New Zealand. Her research areas include computational modelling of a glucose-insulin system, and study and design in the bioengineering field, specifically in medical instrumentation, control, prediction analysis, and rehabilitation engineering.

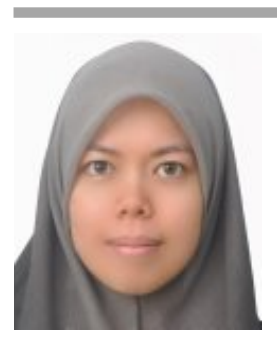

Ummu Kulthum Jamaludin is a senior lecturer with the Faculty of Engineering, Universiti Malaysia Pahang, Malaysia. She received her B.Eng. with Honours in Mechanical Engineering from the University of Canterbury in 2008. This was followed by a $\mathrm{PhD}$ from the same university in 2013. Her areas of expertise include system modeling and dynamics, instrumentation for biomedical engineering and physiological modelling.

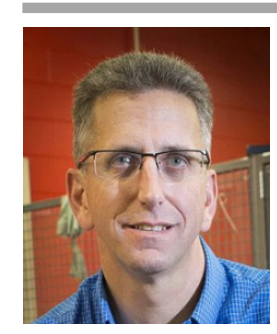

James Geoffrey Chase is a distinguished Professor of UC Mechanical Engineering, who specializes in dynamic systems modelling and control applied to medicine and structures. His pioneering work has real-world impact that ranges from intensive care medicine for babies to earthquake engineering. He received his BSc from Case Western Reserve University, Ohio, in 1986, and his MSc and $\mathrm{PhD}$ from Stanford in 1991 and 1996. He spent six years working for General Motors and a further five years consulting in Silicon Valley, including positions at Xerox PARC, GN ReSound, Hughes Space and Communications, and Infineon Technologies AG before joining the University of Canterbury. His research interests include automatic control, physiological systems dynamics, structural dynamics and vibrations, and dynamic and systems modeling. He was awarded a 2018 University of Auckland Research Excellence Medal, and a 2017 University of Canterbury Research Medal. 